\title{
People in different age groups show different hip-joint morphology
}

\author{
M Müller-Gerbl MD PhD', R Putz MD PhD', R Kenn MD PhD², R Kierse MD PhD² \\ ${ }^{1}$ Anatomische Anstalt der LMU München, and ${ }^{2}$ Radiologische Abteilung der Chirurgischen \\ Klinik Innenstadt München, FRG
}

\begin{abstract}
Summary
It has been suggested that the distribution of the subchondral bone density may be regarded as the expression of the long-term effective stress in a joint, and previous results indicate the regularity of the distribution of subchondral bone density as a function of the passing demands made upon a joint. Computed tomography-osteoabsorptiometry has been developed to visualize the area distribution of subchondral mineralization in the major joints in vivo. The purpose of this study was to display the distribution of subchondral bone density in the acetabular cup of patients of different ages. Computer tomography data files of hip joints of 27 patients (18-89 years) were used. Density ranges, image analysis, and area presentation of the distribution of subchondral mineralization are presented. The maximal subchondral mineralization in young persons is found both in the ventral and dorsal part of the acetabular roof. In older people, however, the densest areas are most often found at the zenith of the acetabulum. These morphological results could be well explained by the experimental results of other authors who found a joint incongruity in young persons with contact areas in the ventral and dorsal part of the acetabulum. With advancing age a decrease in incongruence is found, leading to an increased stress in the dome, i.e. in the area where degenerative changes are often found.
\end{abstract}

\section{Relevance}

CT osteoabsorptiometry is a method of estimating the mineralization pattern of a joint surface in vivo which the conventional anteroposterior radiograph (being a summation picture) cannot provide. Thus a method is available which allows the adaptive capacity of an individual joint to be assessed, and enables progressive observations to be made after a joint has been subjected to abnormal or at least altered mechanical conditions.

Key words: CT osteoabsorptiometry, hip-joint mechanics, subchondral mineralization, biomechanics, functional adaptation

\section{Introduction}

For more than a century the relationship between mechanical stress and the morphology of bone has been accepted as a central point of skeletal research. More recent authors ${ }^{1-3}$ have been able to exemplify this by showing that the instantaneous picture of the morphology of a bone is the expression of the complete loading

Received: 6 February 1992

Accepted: 14 April 1992

Correspondence and reprint requests to: Dr Magdalena Müller-Gerbl, Anatomische Anstalt, Pettenkoferstr. 11, 8000 München 2, Germany

Presented in part at the XIII ${ }^{\text {th }}$ International Congress of Biomechanics, Perth, Australia 9-13 December, 1991.

(C) 1993 Butterworth-Heinemann Ltd 0268-0033/93/020066-07 history to which the tissue has been subjected over a long period of time. Pauwels ${ }^{4.5}$, who continued the work of Wolff ${ }^{6}$, Fick $^{7}$, and Roux ${ }^{8}$ on the interaction between structure and function of the connective and supporting tissues, was also able to establish that the area distribution of the subchondral bone density correlates with the long-term stress acting on a joint. Furthermore, many diagnostic decisions in everyday orthopaedics are not a little dependent upon Pauwels ${ }^{9}$ suggestion that there is a causal relationship between the stress distribution in a joint and the degree of underlying subchondral mineralization. As a wellknown clinical example one may quote the appearance of the subchondral layer of the acetabular roof, which in straight anteroposterior $\mathrm{X}$-rays shows a typical picture in such diseases as, for instance, coxa valga and vara, and which establishes differential diagnosis from the normal hip joint. Indeed, these changes are 
regarded as prearthrotic conditions; although up to now no objective valuation has been possible, since the conventional AP exposure is the result of a summation of images and can give no true picture of the area distribution.

We have therefore employed computed tomography (CT) to develop a method - CT osteoabsorptiometry $(\mathrm{CT} \text { OAM })^{10,11}$ - by means of which it is possible to estimate the area distribution of subchondral mineralization in the larger joints in vivo provided that certain potential sources of error are taken into account. This has made it possible to observe the course of the normal adaptation to the mechanical conditions in a joint - in other words, information over its loading history - and has provided an objective method for assessing its prearthrotic condition.

The aim of this investigation is to demonstrate the pattern of subchondral acetabular density in the normal hip joints of subjects in different age groups.

\section{Methods}

CT data sets (section thickness $4 \mathrm{~mm}$ ) were taken from 27 subjects aged 18-90 years, who had fractured the pelvis. In each case the opposite undamaged side was used for our investigation. Hip joints showing any radiological signs of joint disease were excluded.

\section{CT osteoabsorptiometry ${ }^{10,11}$}

a Basic principles of the method (see Figure 1)

Densitometric evaluation in terms of Hounsfield units was achieved with the Siemens Evados (Siemens, Erlangen, Germany) radiotherapy-planning computer. After magnification of the relevant joint surfaces, conversion into the Sidos-Tele format allows the regions of different density to be distinguished one from another. Then, following input of the postdetermined Hounsfield values, the Sidos-Tele subroutine can produce isodensits, - i.e. contour lines of equal density dividing regions of greater density from those of lesser density. The resulting pictures are then further processed by image-analysing methods and a false colour scheme used to make regions of equal density visible.

In order to obtain a projection of the subchondral density on to the joint surface, the density values of single sections are then measured at a predetermined depth of $1.5 \mathrm{~mm}$, and the results transferred to a contour map of the joint, so that comparative analysis of the distribution of material over the entire joint surface is possible.

b Further development of CT osteoabsorptiometry by means of three-dimensional reconstruction (Figure 2)

This method has been further developed by the introduction of a three-dimensional reconstruction program, by means of which the density distribution of the subchondral mineralization can be directly demonstrated on the computer tomogram. With this program it is possible both to build up the whole articular surface and to indicate the separation of the various density levels. Finally, by employing an image-analysing system, the density grades may be demonstrated in false colours and projected one upon the other.

The advantage of this procedure over earlier methods is that the subchondral density can be projected exactly on to the individual shape of the articular surface of the joint in a much shorter time.

\section{Results}

With regard to both the individual sections (Figure 3) and the density maps of the entire articular surface (Figure 4), zones of maximum bone density were found in the dorsal and ventral regions of the dome of the acetabulum in 14 of the 17 subjects under 60 years of age. In contrast to this, in seven of 10 subjects of more than 60 years, the maximum density appeared in the central region (Table 1). One 65-year-old woman with marked generalized osteoporosis showed the greatest density maximum in the centre of the acetabular dome, together with a significant reduction in the overall degree of mineralization (Figure 4e).

\section{Discussion}

The results obtained from osteoabsorptiometric examination of the hip-joint show that there is a regularly occurring, reproducible pattern of distribution for the degree of subchondral mineralization. The area representation of the density distribution makes it possible to estimate the individual distribution of material easily and quickly. The advantages offered by this procedure, which employes three-dimensional reconstruction, lies in the fact that the accurate projection of the subchondral density onto the individual shape of each joint surface is possible and can, additionally, be done in a much shorter time, in comparison with former methods.

The impact of CT osteoabsorptiometry is particularly well demonstrated by the acetabulum. Although the subchondral sclerosis in both the AP X-rays shown in Figure 5 exhibits the same form ('sourcil'), the CT osteoabsorptiometry reveals fundamentally different distribution patterns (Figures $4 \mathrm{~b}$ and $4 \mathrm{f}$ ). This makes it clear that summation $\mathrm{X}$-rays do not allow a differentiated localization of density differences within a joint surface to be elicited. Our newer method permits information to be obtained not only in the frontal plane, but also in the sagittal plane. In the case of other joint surfaces, such as that of the glenoid cavity or the patella for example, information about the density distribution can only be obtained by means of CT osteoabsorptiometry.

Our results also indicate that age-dependent differences must be taken into account, and that the results obtained from older people cannot necessarily be generalized and applied to younger people; the differences highlighted by comparing the density maps of young and old hip joints suggests that the mechanical 

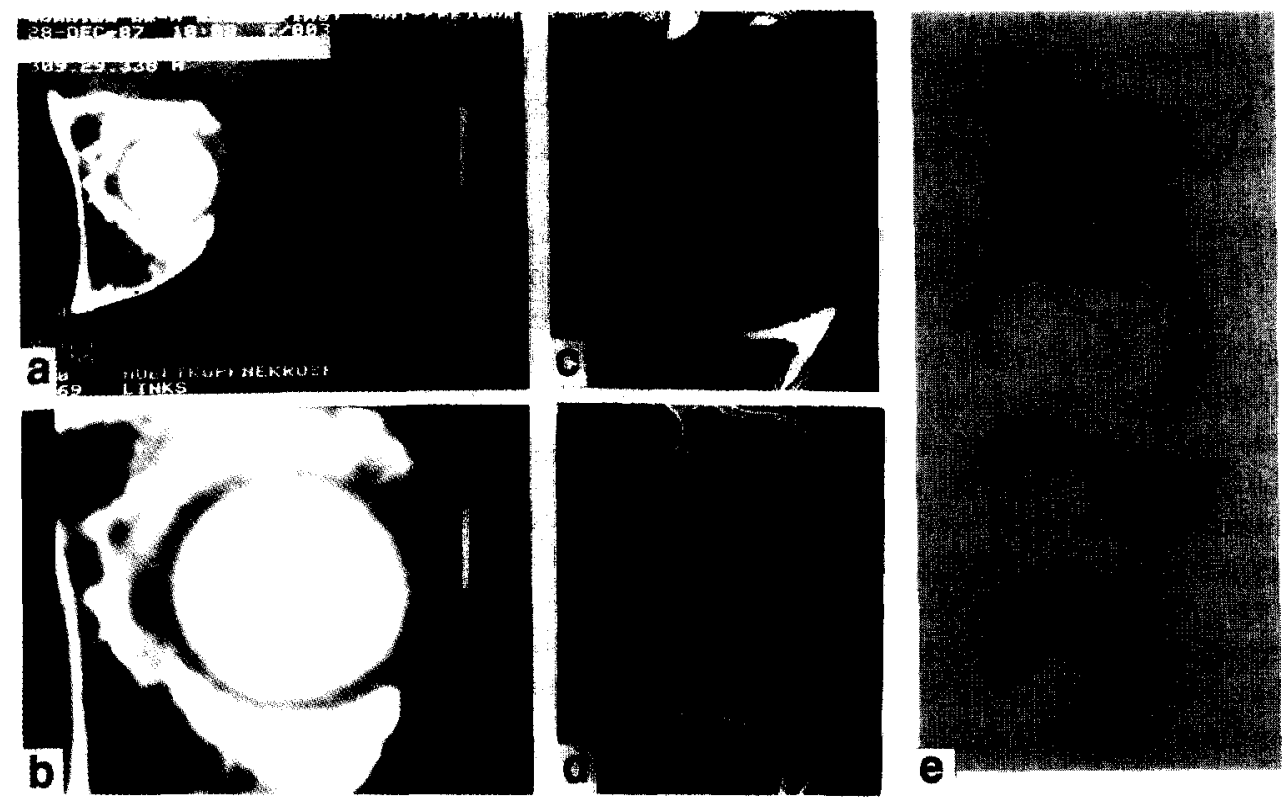

Figure 1. CT osteoabsorptiometry of a right hip joint. a. Transverse section. b. Magnification of the hip-joint region. c. Isodensits in subchondral bone: acetabulum and femoral head. d. False colour demonstration of the various density zones. e. Production of a surface density map from individual sections.
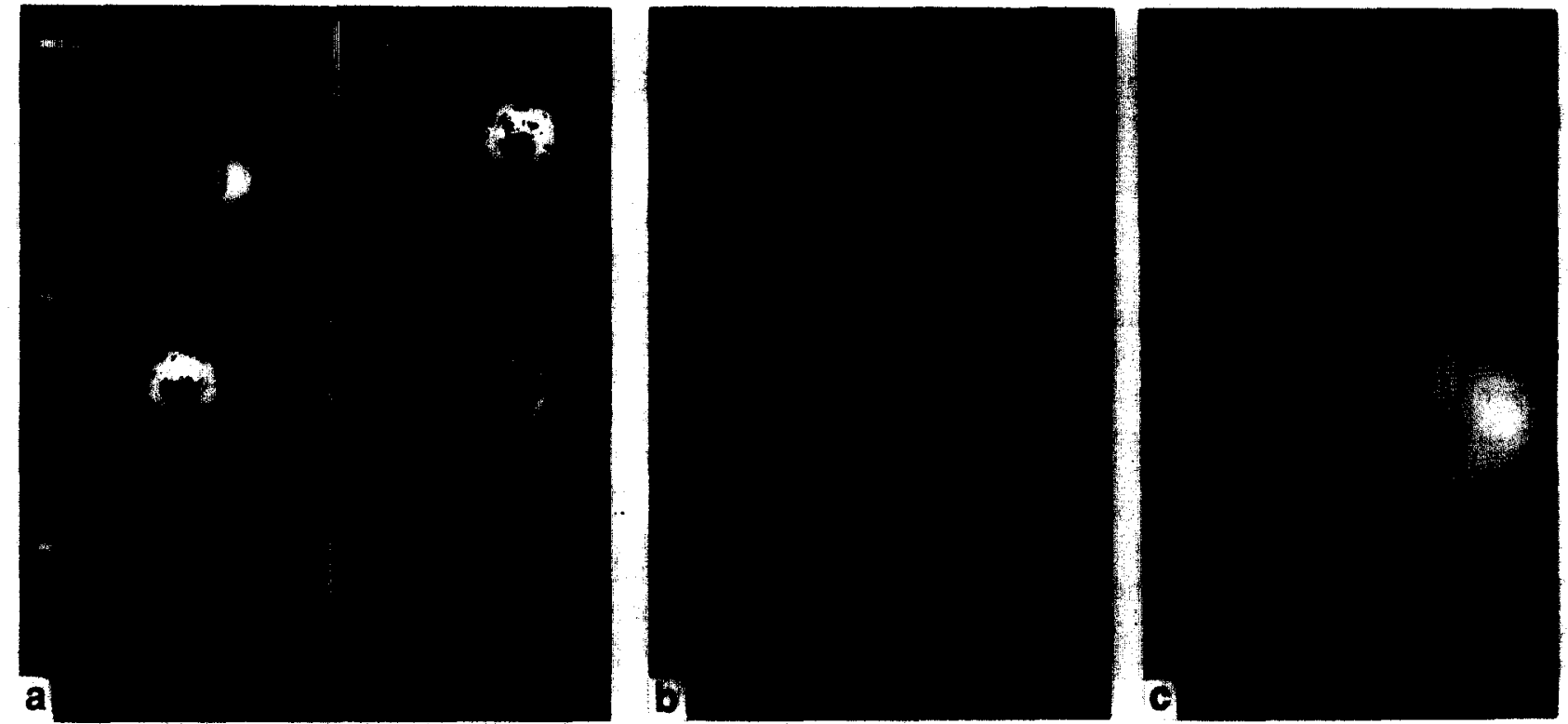

Figure 2. CT osteoabsorptiometry using a 3-D program. a. Pictures of the whole articular surface of the acetabulum and of the individual density levels, generated from the computer tomograms themselves (lateral view). b. Method of false colour demonstration and projected superimposition. c. Results after using image analyser.
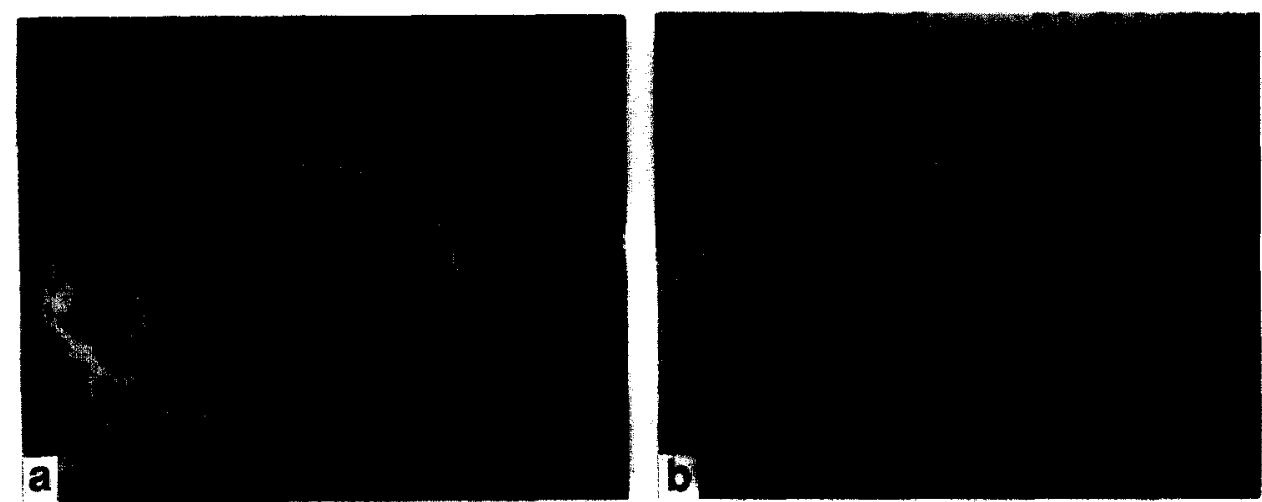

Figure 3. Density distribution in individual sections, parallel to the plane of inlet of the acetabulum. a. a 36-year-old woman. b. an 82-year-old woman. 
a
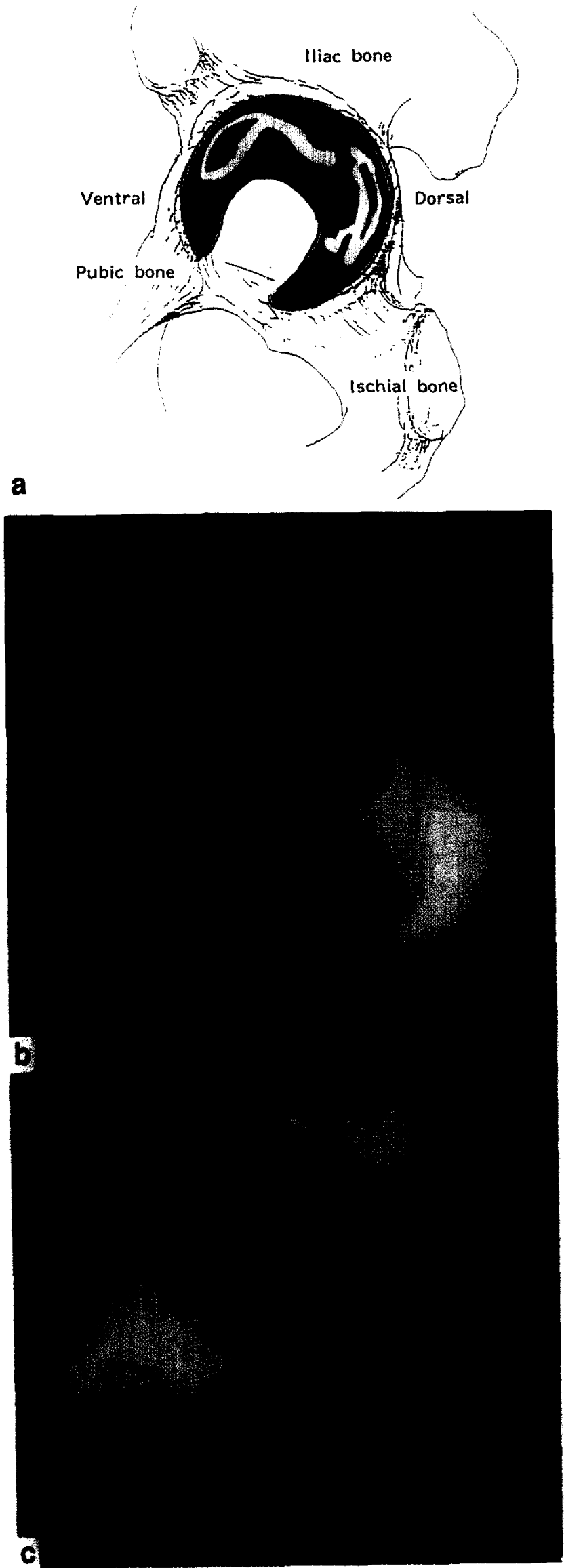
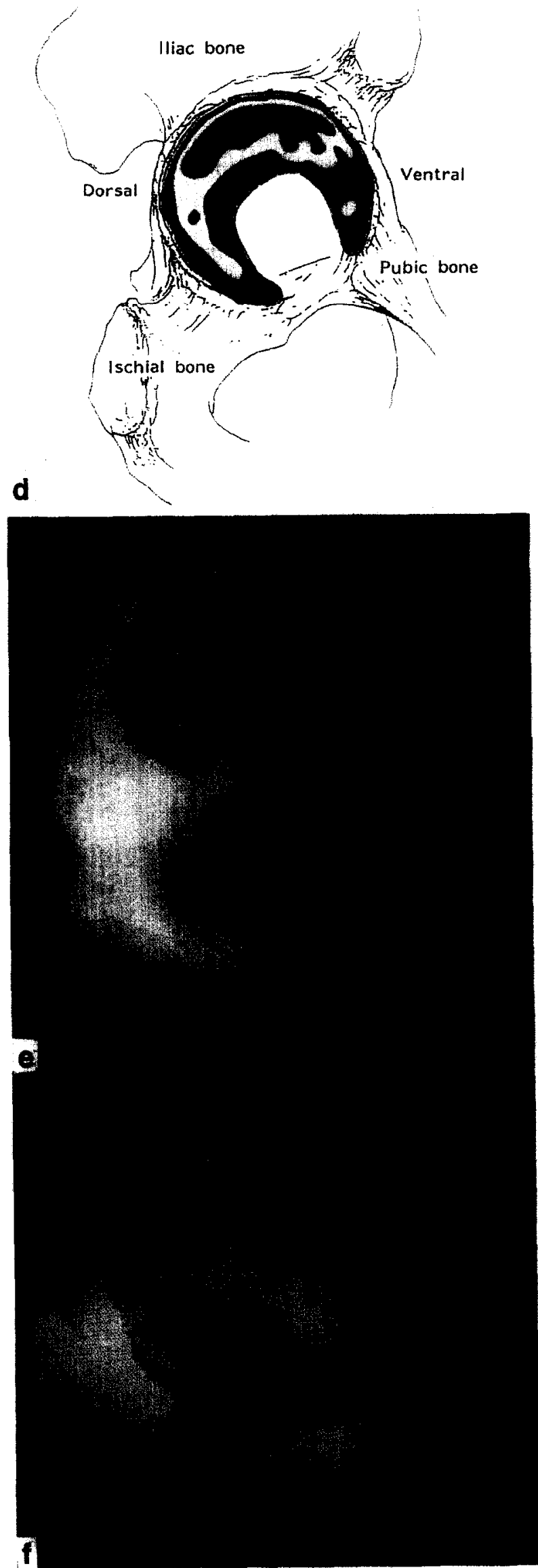

Figure 4. Density maps of the facies lunata of the hip-joint (lateral view). a, a 36-year-old man. b, a 18-year-old woman (3D CT osteoabsorptiometry). c, a 45-year-old man. d, a 85-year-old man. e, a 65-year-old woman (3DCT). f, a 68-year-old woman (3DCT). 
Table 1. Correlation between the localization of the maxima of subchondral bone density and age $(n=27)$

\begin{tabular}{rcc}
\hline & Central & Ventral/dorsal \\
\hline $18-59$ years & 3 & 14 \\
$60-90$ years & 7 & 3 \\
\hline
\end{tabular}

situation in these joints is dependent upon the age of the subject. The results of an X-ray densitometry investigation ${ }^{12}$ on six hip sockets taken from dissectingroom material are, although based on a smaller number of cases, in agreement with our own findings in the living subject.

The significance of the distribution pattern of subchondral mineralization for the mechanics of a joint becomes all the more plain if our findings on the facies lunata are placed in context with the results and interpretations of other authors.

In younger people we found that the zone of maximum bone density is not in the middle, but at the front and back of the joint, whereas in older persons a different pattern with a central maximum is seen. Bullough $^{13}$ et al's investigations suggest a possible explanation of this apparent age-dependent difference, since in the unloaded condition he found an incongruence of the bones in young subjects. However, this incongruence decreases with advancing age. A related study into the contact areas of the hip joint ${ }^{14}$ accordingly demonstrated in young people, in the case of small loads, the existence of contact zones at the back and front of the acetabulum, which were independent of the position of the joint. In older subjects, on the other hand, the contact zone is found in the dome of the socket. In their experiments on the size and localization of contact areas, Greenwald and Haynes ${ }^{15}$ found deficient contact zones in the dome in $83 \%$ of subjects under 60 years old, and only $36 \%$ in those over 60 . These numbers agree quantitatively with our own morphological results (see Table 1).

According to the investigations of Miyanaga ${ }^{16}$ et al. on loading at the hip joint, an active load on the joint corresponding to about $60 \%$ of the body weight brings about complete approximation of the articular surfaces, with a correspondingly greater area of contact and less pressure, even in primarily incongruent joints. This, as comprehensive experiments on the load-deformation behaviour of the cartilage and underlying bone have demonstrated, is due to the fact that the hip-joint as a whole retains its viscoelasticity.

A model (Figure 6) of the influence of its geometry on the stress received by a joint put forward by Bullough $^{17}$ has convincingly illustrated the situation in both young and old subjects. In joints of type 1 , which are to be found in older people, the contact area under
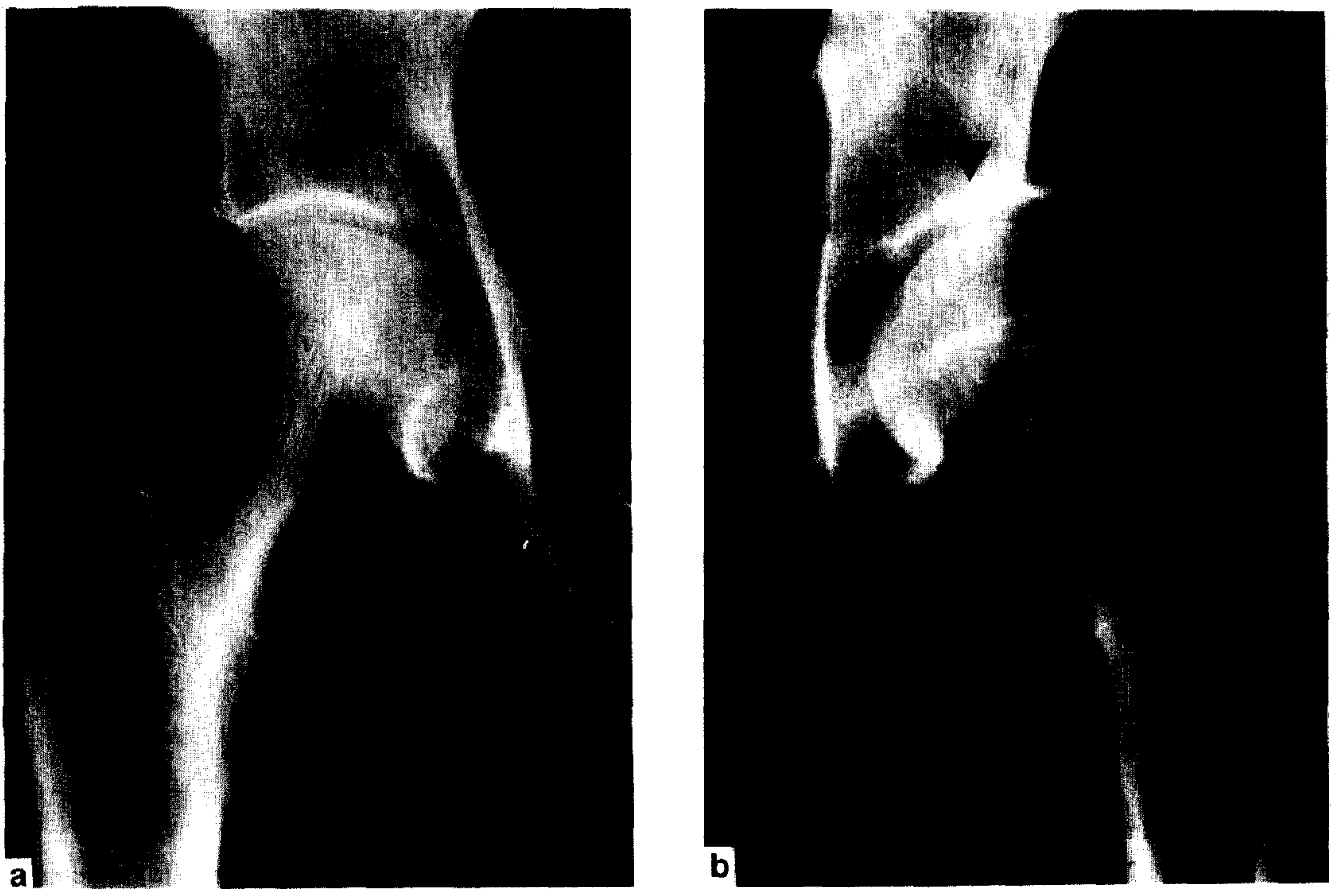

Figure 5. AP X-rays of the hip joint of the same persons as in Figure 4. In spite of the different density patterns by CT osteoabsorptiometry the shape of the subchondral layer of the acetabulum (arrows) show no difference in both $X$-rays. a, 18-year-old woman (same patient as in Figure 4b). b, 68-year-old woman (same patient as in Figure 4f). 

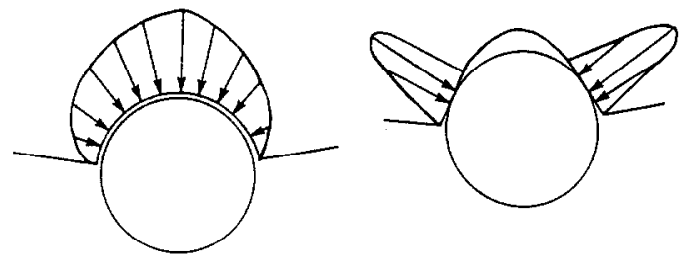

Unloaded

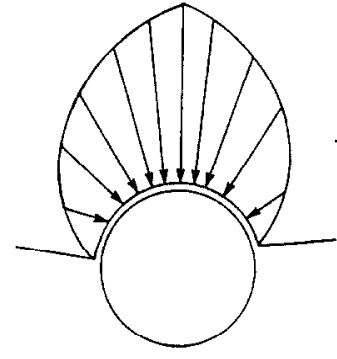

a

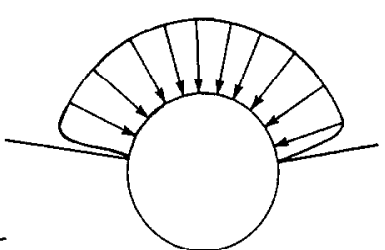

Loaded
Figure 6. Model of the stress distribution in a ball-andsocket joint. a. A congruent joint (situation in older people). b. An incongruent joint (situation in younger people) (redrawn after Bullough ${ }^{17}$ ).

increasing load will become narrower, and the stress correspondingly greater. In unloaded joints of type 2 with primary incongruence, the contact zones are found only on the periphery. If the load increases, the viscoelastic deformation of the articular cartilage and underlying bone leads to an increase in the size of the contact area, and thereby a smaller pressure, so that this joint, as well as possessing greater stability, is best able to reduce load and to produce the most uniform load distribution. In addition to this, the incongruence present in the unloaded or lightly loaded joint facilitates fluid exchange between the articular cartilage and synovial fluid, thereby ensuring optimal nutrition for the articular cartilage. With permanent complete congruence of the joint surfaces, as in type 1, the absence of a cleft greatly influences and limits the metabolic activity of the chondrocytes.

A quantitative comparison of the demands made upon the joint by different actions, and the number of times that these actions are performed during the day, made by Marsden and Montgomery ${ }^{18}$ led to the conclusion that only for $5-25 \%$ of the day are the loads sufficiently strenuous to bring about complete apposition of the articular surfaces. This leads to increased local loading on the anterior and posterior parts of the acetabulum, but if continuous, it is also responsible for relative underloading of the central part of acetabular cup. Age-dependent degenerative changes in the cartilage initially appear in unloaded rather than loaded parts of the joint ${ }^{14.19 .20}$. In the hip joint this amounts to a more or less triangular area of the acetabular dome, the basc of which comes to lie at the outer margin of the socket ${ }^{14}$. These changes range from a reduced proteoglycan content of the cartilage to fibrillation of the cartilage $^{13}$. A proteoglycan deficiency, which can result from many causes, may also be due to a reduction in their use or to a prolonged reduction of the load, a fact which has up to now been largely ignored. In the same way that immobilized bones or muscles atrophy, a reduction in the demands made upon cartilage can result in reduced proteoglycan synthesis by the chondrocytes. Attention has been drawn to this kind of positive correlation between regions where degeneration is common, and those where the stress is usually minimal, in the elbow joint ${ }^{21}$ and in the knee joint ${ }^{22}$. These degenerativc changes in areas of cartilage do not impair the normal functioning of the joint, so long as the incongruence remains. The incongruence maintains the environment of the cartilage in proper condition, but it is lost in older people. The result is an altered distribution of intra-articular pressure, so that previously unloaded regions of the joint are subjected to heavy pressure. These regions - reduced to a condition of chondromalacia by long-standing disuse - must now carry a considerable stress for which they are not adapted. An irreversible process of cartilaginous degeneration then sets in, which is in turn accelerated by poor nutrition. In other words, the long-term lack of usage in certain areas of the cartilage, together with an increasing loss of the articular incongruence, can be identified as important factors in bringing about arthrosis of mechanical origin in older people.

It seems therefore reasonable to accept that the components of a normal hip joint are so exactly fitted together that favourable conditions are provided, both for lubricating and nourishing the normally loaded cartilage, as well as for the transmission and distribution of load.

In our opinion the morphological findings on the subchondral mineralization of the acetabulum exactly reflect the mechanical relationships involved, so that the density pattern seems to be capable of providing an ideal interpretation of the individual loading history of the joint. CT osteoabsorptiometry has therefore also provided us with a method of testing the results derived experimentally from dissecting-room specimens, and verifying their validity in the living subject.

\section{References}

1 Carter DR. Mechanical loading histories and cortical bone remodelling. Calcif Tissue Int 1984; 36: \$19-S24

2 Cowin SC. Wolff's law of trabecular architecture at remodelling equilibrium. J Biomech Eng 1986; 108: 83-8

3 Frost HM. Intermediary Organization of the Skeleton. Boca Raton, Florida: CRC Press, 1986: 1-11

4 Pauwels F. Gesammelte Abhandlungen zur funktionellen Anatomie des Bewegungsapparates. Berlin-HeidelbergNew York: Springer, 1965

5 Pauwels F. Biomechanics of the Locomotor Apparatus. Berlin-Heidelberg-New York: Springer, 1980

6 Wolff J. Das Gesetz der Transformation der Knochen. Berlin: Verlag A. Hirschwald, 1892

7 Fick R. Handbuch der Anatomie des Menschen. Vol. II Spezielle Gelenk- und Muskelmechanik. Jena: Fischer, 1911

8 Roux W. Anpassungslehre, Histomechanik und Histochemie. Mit Bemerkungen über die Entwicklung und Formgestaltung der Gelenke. Virchows Arch Path Anat 1912; 209: $168-209$ 
9 Pauwels F. Atlas zur Biomechanik des gesunden und kranken Hüfte. Berlin-Heidelberg-New York: Springer, 1973

10 Müller-Gerbl M, Putz R, Hodapp N, Schulte E, Wimmer B. Computed tomography-osteoabsorptiometry for assessing the density distribution of subchondral bone as a measure of long-term mechanical adaptation in individual joints. Skeletal Radiol 1989; 18: 507-12

11 Müller-Gerbl M, Putz R, Hodapp N, Schulte E, Wimmer B. Computed tomography-osteoabsorptiometry: a method of assessing the mechanical condition of the major joints in a living subject. Clin Biomech 1990; 5: 193-8

12 Oberländer W. Die Beanspruchung des menschlichen Hüftgelenks. V. Die Verteilung der Knochendichte im Acetabulum. Z Anat Entw-gesch 1973; 140: 367-84

13 Bullough P, Goodfellow J, Greenwald AS, O'Connor J. Incongruent surfaces in the human hip joint. Nature 1968; 217: 1290

14 Bullough P, Goodfellow J, O'Connor J. The relationship between degenerative changes and load-bearing in the human hip. J Bone Joint Surg 1973; 55B: 746-58

15 Greenwald AS, Haynes DW. Weight-bearing areas in the human hip joint. $J$ Bone Joint Surg 1972; 54B: 157-63
16 Miyanaga Y, Fukubayashi T, Kurosawa H. Contact studies of the hip joint. Arch Orthop Trauma Surg 1984; 103: $13-17$

17 Bullough P. The geometry of diarthrodial joints, its physiologic maintenance, and the possible significance of age-related changes in the geometry-to-load distribution and the development of osteoarthritis. Clin Orthop 1981; 156: 61-6

18 Marsden JP, Montgomery SR. Plantar power for arm prosthesis using body weight transfer. Conference on human locomotor engineering at the University of Sussex. London: Institution of Mechanical Engineers September 7-10, 1971

19 Harrison MHM, Schajowicz F, Trueta J. Osteoarthritis of the hip: a study of the nature and evolution of the disease. J Bone Joint Surg 1953, 35B: 598-626

20 Byers PD, Contepomi CA, Farkas TA. A post mortem study of the hip joint. Ann Rheum Dis 1970; 29: 15-31

21 Goodfellow JW, Bullough PG. The pattern of ageing of the articular cartilage of the elbow joint. J Bone Joint Surg 1967; 49B: $175-81$

22 Goodfellow JW, Hungerford D, Zindel M. Chondromalacia patellae. Orthopaedics: Oxford Volume 1971; 4: 111 